\title{
AVALIAÇÃO DA ACURÁCIA POSICIONAL EM DADOS ESPACIAIS UTILIZANDO TÉCNICAS DE ESTATÍSTICA ESPACIAL: PROPOSTA DE MÉTODO E EXEMPLO UTILIZANDO A NORMA BRASILEIRA.
}

\author{
Assessment of positional accuracy in spatial data using techniques of spatial \\ statistics: proposal of a method and an example using the Brazilian standard
}

\author{
Afonso de Paula dos Santos ${ }^{1}$ \\ Dalto Domingos Rodrigues ${ }^{1}$ \\ Nerilson Terra Santos ${ }^{2}$ \\ Joel Gripp Junior ${ }^{1}$
}

\begin{abstract}
${ }^{1}$ Dept. Eng ${ }^{\mathrm{a}}$ Civil - Setor de Eng ${ }^{\mathrm{a}}$ Agrimensura e Cartográfica - Universidade Federal de Viçosa - UFV Av. P.H. Rolfs, s/n, Campus Universitário, CEP 36570-900, Viçosa, Minas Gerais, Brasil.

2 Dept. Estatística - Universidade Federal de Viçosa - UFV - Av. P.H. Rolfs, s/n, Campus Universitário, CEP 36570-900, Viçosa, Minas Gerais, Brasil.
\end{abstract}

Email:afonso.santos@ufv.br; dalto@ufv.br; nsantos@ufv.br; jgripp@ufv.br;

\section{Resumo:}

Este trabalho destaca a importância da utilização de técnicas simples de estatística espacial no controle de qualidade posicional em dados espaciais. Para tanto, são apresentados métodos de análise do padrão de distribuição espacial de dados pontuais, bem como a análise de tendências na amostra de discrepâncias posicionais. Para avaliar a distribuição espacial de pontos, utilizaram-se os métodos do Vizinho Mais Próximo e da Função K de Ripley. Já para análise de tendência, utilizou-se a média direcional dos vetores de discrepâncias aliada à variância circular. Neste sentido, propõe-se uma metodologia para o controle de qualidade posicional em dados espaciais abrangendo desde o planejamento da amostra e avaliação de seu padrão de distribuição espacial, a aplicação de teste de tendências analisando a normalidade dos dados, e a classificação em relação a uma determinada norma de acurácia posicional. Para o experimento prático, avaliou-se uma ortoimagem gerada a partir de uma cena do sensor PRISM do satélite ALOS. Os resultados mostram que a ortoimagem é acurada para a escala de 1:25.000, sendo classificada como classe A conforme padrão brasileiro de acurácia posicional, não apresentando tendências nas coordenadas. Como principal contribuição deste trabalho, tem-se a incorporação de técnicas de estatística espacial no controle de qualidade cartográfica.

Palavras-chaves: Controle de Qualidade, Acurácia Posicional, Estatística Espacial, Cartografia.

\begin{abstract}
:
This paper presents the importance of simple spatial statistics techniques applied in positional quality control of spatial data. To this end, Analysis methods of point data spatial distribution pattern are presented, as well as bias analysis in the positional discrepancies samples. To
\end{abstract}


evaluate the points spatial distribution Nearest Neighbor and Ripley's K function methods were used. As for bias analysis, the average directional vectors of discrepancies and the circular variance were used. A methodology for positional quality control of spatial data is proposed, in which includes sampling planning and its spatial distribution pattern evaluation, analyzing the data normality through the application of bias tests, and positional accuracy classification according to a standard. For the practical experiment, an orthoimage generated from a PRISM scene of the ALOS satellite was evaluated. Results showed that the orthoimage is accurate on a scale of 1:25,000, being classified as Class A according to the Brazilian standard positional accuracy, not showing bias at the coordinates. The main contribution of this work is the incorporation of spatial statistics techniques in cartographic quality control.

Keywords: Quality Control, Positional Accuracy, Spatial Statistics, Cartography.

\section{Introdução}

O principal objetivo da avaliação da qualidade de dados espaciais é a definição das finalidades para as quais eles podem ser destinados. A cartografia produz modelos da realidade, e estes são utilizados para tomar decisões. Assim, um maior nível de controle de qualidade em uma base de dados espaciais pode propiciar um maior nível de acerto na tomada de decisões.

Atualmente, é crescente a utilização de dados espaciais nas mais variadas áreas de conhecimento, tais como: ciências e tecnologias, saúde, segurança, planejamento, turismo, dentre outros. Vivencia-se uma revolução geoespacial, em que a tecnologia utilizada no mapeamento se desenvolve com espantosa velocidade: cresce a cada dia a quantidade de dados espaciais disponíveis gratuitamente, de sensores orbitais, métodos e algoritmos. Além disto, softwares cada vez mais amigáveis encontram-se disponíveis para o uso de todos.

Desta forma, de acordo com Galo e Camargo (1994), esta evolução tecnológica eleva proporcionalmente o número de usuários não especialistas em mapeamento. Assim, em função desta condição, muitas vezes o cuidado com a qualidade posicional é esquecido.

Nesse contexto, é importante destacar que o conceito de qualidade se refere ao conjunto de características de um produto em satisfazer as necessidades, explícitas e implícitas, do usuário (ISO 2002). Na área cartográfica, a avaliação da qualidade de dados espaciais deve considerar diversos elementos como acurácia posicional, acurácia temporal, completude, consistência lógica e temporalidade, o que torna a análise um tanto quanto complexa. Segundo Ariza e Atkinson (2008), a acurácia posicional e a consistência lógica são os elementos mais focados nos institutos de mapeamentos. A acurácia posicional refere-se a quão próxima a posição de um dado espacial está em relação à sua realidade no terreno (Nogueira Jr. 2003; Ariza 2002).

Segundo Aronoff (1989), o importante em qualquer levantamento espacial não é eliminar, mas gerenciar a incerteza inerente ao processo, seja na aquisição de novas informações ou simplesmente sua conversão. A incerteza, neste contexto, pode ser definida como alguma discrepância entre as situações descritas nos dados espaciais e a realidade (Arbia et al. 1998).

Assim, para avaliar a acurácia posicional de dados espaciais utiliza-se de uma amostra de checagem. As posições do dado espacial são confrontadas com as posições da amostra de checagem, obtendo-se, assim, um conjunto de discrepâncias posicionais. Estas discrepâncias são utilizadas para a classificação da acurácia posicional do dado espacial de acordo com alguma 
norma.

Atualmente, há vários padrões para se proceder à avaliação da qualidade posicional de dados espaciais, como: ISO 19114 - Informação Geográfica - Procedimentos de Avaliação da Qualidade; National Map Accuracy Standard (NMAS) - EUA; Engineering Map Accuracy Standard (EMAS) - EUA; Accuracy Standards for Large Scale Maps (ASLSM) - EUA; National Standard for Spatial Data Accuracy (NSSDA) - EUA; NDATAVLBD 1983 - Germany, Estándar Nacional para la Precisión de los Datos Espaciales (ICONTEC) Colombia 2001, STANAG 2215 - Standard from North Atlantic Treatment Organization (NATO). No Brasil, a avaliação pode ser feita a partir do Padrão de Exatidão Cartográfica (PEC) definido no Decreto-Lei $\mathrm{n}^{\circ}$. 89.817 de 1984, que regulamenta a classificação dos produtos cartográficos quanto à sua acurácia posicional (Brasil 1984). Em 2010, foram publicadas as Especificações Técnicas de Aquisição de Dados Geoespaciais Vetoriais (ET-ADGV), documento este ligado à Infraestrutura Nacional de Dados Espaciais (INDE), que pode ser aliado ao Decreto-lei $n^{\circ} 89.817$ para uma avaliação mais rigorosa de produtos cartográficos digitais.

A diversidade de critérios e padrões de acurácia posicional, conforme descritos em trabalhos de Ariza e Atkinson (2008) e Nero (2005), mostra que o assunto precisa ser abordado com grande rigor, pois o uso de diferentes critérios pode conduzir a diferentes classificações para um mesmo produto.

Um problema recorrente na avaliação da qualidade posicional em algumas normas é a falta de análise efetiva da distribuição dos pontos amostrados. Atualmente, normas como ASLMS, NSSDA, STANAG e ICONTEC contemplam metodologias empíricas para a determinação do padrão de distribuição espacial da amostra. Nas normas que não contemplam metodologias, quando existe alguma orientação, esta se dá apenas no sentido de que a amostra deve ser bem distribuída na área de estudo, o que faz com que a maioria das avaliações em relação à distribuição espacial da amostra seja realizada subjetivamente, de forma visual.

Assim, uma amostra de checagem com o padrão de distribuição espacial do tipo agrupado pode comprometer a avaliação da acurácia posicional deste dado espacial. Se o padrão de amostragem estiver agrupado apenas em certa área da região, a avaliação da acurácia posicional não pode ser estendida para toda a área de abrangência do dado espacial, mas apenas nesta área compreendida pelo conjunto de pontos agrupados. Logo, técnicas de estatística espacial, como métodos do Vizinho Mais Próximo e a Função $K$ de Ripley, podem ser bastante úteis para a análise das amostras quanto à distribuição espacial das mesmas.

Além da distribuição da amostra, no controle de qualidade de dados espaciais é de fundamental importância a análise de tendências. Ao se utilizar um dado tendencioso, com erros sistemáticos, a integração entre dados fica impossibilitada em um banco de dados espaciais, podendo gerar análises, decisões e produtos que não condizem com a realidade de campo. Atualmente, é fácil encontrar no mercado de trabalho produtos gerados com erros sistemáticos, sendo a principal causa a falta de controle nas várias etapas da produção cartográfica. Erros em transformações de sistemas de referenciais geodésicos, erros de projeção, confusões com metadados, utilização de equipamentos não retificados e equações ou procedimentos mal utilizados são exemplos de elementos que podem inserir tendências nos dados.

A maioria das avaliações de acurácia posicional que analisam tendências utilizam o teste de hipóteses t de Student, conforme descrito em Merchant (1982). Este teste necessita que a amostra de discrepâncias posicionais tenha distribuição normal. Entretanto, podem ocorrer casos em que a distribuição não seja normal. Nestas hipóteses, a solução é utilizar simples estatísticas espaciais, que retornam a média direcional das discrepâncias e a variabilidade desta média, podendo assim, dizer se o dado espacial está com presença ou não de tendência. 
Sendo assim, o objetivo principal deste trabalho é incorporar técnicas simples de estatística espacial no controle de qualidade posicional em dados espaciais. Considerando esse objetivo geral, destacam-se os seguintes objetivos específicos:

- Apresentar alguns métodos, provenientes da estatística espacial, que podem ser utilizados para analisar o padrão de distribuição espacial dos pontos amostrados, bem como a presença de tendências em dados espaciais.

- Definir uma metodologia para a avaliação da acurácia posicional com o uso da estatística espacial e aliar esta metodologia ao padrão de acurácia posicional brasileiro, para aplicação prática em uma ortoimagem ALOS/PRISM;

No âmbito das informações espaciais, a qualidade está intimamente ligada à finalidade de uso do produto. Conhecendo a utilização do produto cartográfico e as incertezas admissíveis, é possível exigir dos produtores uma especificação de qualidade, tanto para o produto final quanto para cada etapa de produção, o que trará ganho em termos de custo/benefício (Nero 2005). Tais produtos cartográficos são o alicerce de qualquer planejamento, em termos territoriais e de engenharia, que se queira realizar. Logo, se a base cartográfica não atende um padrão de qualidade aceitável, todo o processo seguinte estará comprometido.

Portanto, percebe-se o quão importante é o desenvolvimento de metodologias mais rigorosas para a avaliação da acurácia posicional de dados espaciais. Neste sentido, o propósito deste artigo é apresentar uma metodologia que incorpore simples técnicas de estatística espacial para a avaliação da distribuição espacial da amostra de checagem, bem como a análise de tendências em dados espaciais.

\section{Determinação do padrão de distribuição espacial dos dados pontuais}

Em uma avaliação de acurácia posicional, planejam-se pontos de checagem de modo a abranger toda a área de estudo. Desta maneira, conhecer o padrão de distribuição espacial dos pontos é de fundamental importância para escolher qual método de avaliação da acurácia posicional será utilizado, ou até mesmo refazer a amostragem dos pontos de checagem para obter determinado padrão de distribuição espacial. Este procedimento proporciona otimização de tempo e custos no processo de avaliação da acurácia posicional.

Com o uso de técnicas de análises espaciais é possível verificar se o padrão de distribuição dos pontos é agrupado, disperso ou aleatório. Estas técnicas começaram a ser desenvolvidas na década de 1950, sendo utilizada em diferentes áreas do conhecimento, principalmente em ecologia e ciências florestais.

Os métodos mais simples de análise do padrão de distribuição espacial de pontos são baseados, principalmente, na contagem de pontos em uma determinada região da área de estudo ou na distância entre o ponto e o vizinho mais próximo. Estes métodos conseguem apenas detectar o padrão espacial nas escalas particulares em que os dados foram coletados (Dalmaso et al. 2013). Para aprofundar na solução do problema, recorre-se à estatística espacial utilizando técnicas como o Vizinho Mais Próximo de altas ordens e a Função $K$ de Ripley (Ripley 1977; Wong e Lee 2005). Estes métodos, que permitem a análise multi-escala da distribuição espacial de pontos, são chamados de funções de segunda ordem (Jones et al. 1996). Neste artigo, serão descritas a estatística do Vizinho Mais Próximo e a Função $K$. 


\subsection{Estatística do Vizinho mais Próximo de alta ordem}

Este método compara a distância média entre os vizinhos mais próximos com um conjunto de pontos que têm um padrão definido teoricamente. Normalmente, o método do Vizinho Mais Próximo é utilizado apenas na primeira ordem $(k=1)$, mas existem variações como a segunda ordem ou $k$ ordens. A segunda ordem $(k=2)$ utiliza a segunda menor distância entre os outros pontos e assim sucessivamente para as outras ordens.

Os passos para realizar a análise de vizinho mais próximo, para $k$ ordens, são:

1. Determinar as distâncias de cada ponto a todos os outros pontos, a partir das coordenadas observadas;

2. Selecionar as $k$ menores distâncias observadas;

3. Obter a média das distâncias observadas no passo anterior;

4. Calcular o índice $R$ para a ordem $k$, que é a razão da média observada da distância ao $k$ vizinho mais próximo com a média esperada para uma distribuição aleatória dos pontos:

a. Se a média for igual ao padrão $(R=1)$, a amostra segue o padrão de distribuição aleatório;

b. Se a média for menor que o padrão $(R<1)$, a amostra segue o padrão de distribuição agrupado;

c. Se a média for maior que o padrão $(R>1)$, a amostra segue o padrão de distribuição disperso;

O índice $R$ pode ser matematicamente calculado pela Equação 1 e visualmente interpretado conforme a Figura 1. A Tabela 1 apresenta os valores das constantes $(\gamma 1)$ para o cálculo da média esperada para uma distribuição aleatória dos pontos em relação à ordem $k$ utilizada.

$$
R(k)=\frac{R_{o b s}(k)}{R_{\text {exp }}(k)}
$$

onde

$$
R_{o b s}(k)=\frac{\sum_{i=1}^{n} d v_{i}(k)}{n}
$$

$$
R_{\text {esp }}(k)=\gamma 1_{(k)} \sqrt{\frac{A}{n}}
$$

sendo:

$R_{o b s}(k)$ : média observada das distâncias de cada ponto ao seu k vizinho mais próximo;

$R_{\text {esp }}(k)$ : média esperada das distâncias entre os k vizinhos mais próximos para a distribuição aleatória;

$d v_{i}(k)$ : distância de um ponto i ao seu k vizinho mais próximo;

$n$ : número de pontos;

$A$ : área da região em estudo.

Para inferir se o índice R é estatisticamente igual ao valor da distribuição aleatória aplica-se o 
teste Z, onde na hipótese nula admite-se que o padrão da distribuição espacial dos dados siga o padrão aleatório. De acordo com Wong e Lee (2005), se a estatística $Z$ calculada para a ordem $k$ $\left(Z_{R}(k)\right)$ através da Equação 04 for maior que o valor tabelado para $Z\left(Z_{t a b}\right)$, rejeita-se a hipótese nula.

Tabela 1: Constantes para o cálculo da estatística do vizinho mais próximo.

\begin{tabular}{c|c|c}
\hline Ordem $(\mathrm{k})$ & $\gamma 1(k)$ & $\gamma 2(k)$ \\
\hline 1 & 0.5000 & 0.2613 \\
\hline 2 & 0.7500 & 0.2722 \\
\hline 3 & 0.9375 & 0.2757 \\
\hline 4 & 1.0937 & 0.2775 \\
\hline 5 & 1.2305 & 0.2784 \\
\hline 6 & 1.3535 & 0.2789 \\
\hline
\end{tabular}

$$
\begin{gathered}
Z_{R}(k)=\frac{R_{o b s}(k)-R_{\exp }(k)}{S E_{r}(k)} \\
S E_{r}(k)=\gamma 2_{(k)} \sqrt{\frac{A}{n^{2}}}
\end{gathered}
$$

onde, a variável $S E_{r}(k)$ representa o erro padrão da diferença entre as distâncias médias observadas e as esperadas entre os vizinhos mais próximos para a ordem $k$.

Esta técnica é bastante sensível ao valor da área, sendo que pequenas mudanças neste valor podem alterar consideravelmente os resultados. Por isto, tal técnica é apropriada para a comparação de vários conjuntos de dados em uma área de estudos fixa.

A proposta de se utilizarem várias ordens na análise de vizinhos mais próximos é compreender como o padrão de distribuição espacial da amostra se comporta em nível local ou regional. A primeira ordem realiza uma análise puramente local, enquanto ordens maiores permitem detectar padrões heterogêneos ocorrendo em diferentes escalas. Ao analisar o padrão de distribuição espacial da Figura 2.a tem-se: $1^{\mathrm{a}}$ ordem e $2^{\mathrm{a}}$ ordem apresentando o padrão agrupado (já que as distâncias entre os pontos mais próximos são pequenos em comparação com a área dos dados), e a $3^{\mathrm{a}}$ ordem apresentando o padrão disperso (já que os pontos próximos estão distantes). Logo, pode-se inferir que o padrão de distribuição espacial da amostra da Figura 2.a é localmente agrupado e regionalmente disperso.

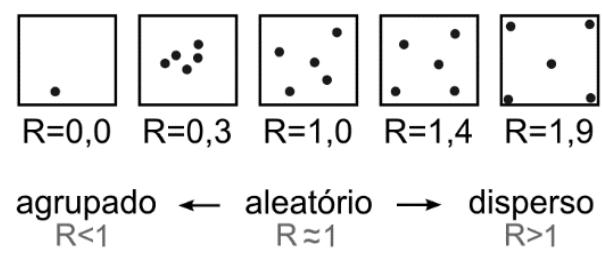

Figura 1: Interpretação do índice R na detecção do padrão de distribuição espacial de dados pontuais. 


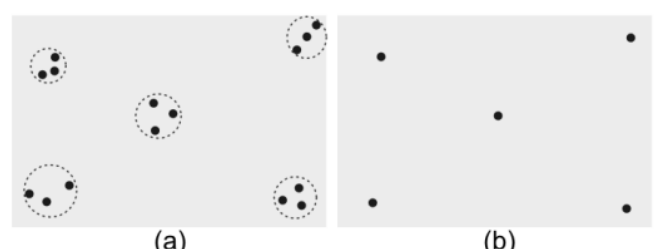

Figura 2: Amostra (a) com padrão localmente agrupado e regionalmente dispersa; Amostra (b) local e regionalmente dispersa.

No controle de qualidade posicional, uma amostra como a da Figura 2.a significa que há vários pontos próximos entre si, mas que, para a área de cobertura, a amostra está bem distribuída por toda sua extensão, apresentando padrão disperso. Baseando-se na primeira lei da geografia que diz que "todas as coisas são relacionadas, mas coisas mais próximas são mais relacionadas", pode-se inferir que a incerteza posicional presente nos grupos de pontos agrupados (representados na Figura 2.a pelo círculo pontilhado) são semelhantes. Esta informação de padrão agrupado localmente diz que a amostra poderia ser reduzida deixando apenas um ponto em cada grupo de pontos, como apresentado pela Figura 2.b.

\subsection{Função $K$ de Ripley}

A Função $K$ de Ripley, quando comparada à análise do vizinho mais próximo, é mais robusta e complexa. Esta técnica avalia se a distribuição espacial se mantém constante em diferentes escalas, ou seja, sob um intervalo de distâncias.

Segundo Anjos et al. (2004), a Função $K$ é uma função de densidade de probabilidade que considera a variância de todas as distâncias entre todos as localizações dos pontos. O método funciona criando círculos de raio $h$, centralizados em cada ponto, sendo quantificado o número de pontos que se localizam dentro do círculo.

Segundo Wong e Lee (2005), a análise da distribuição espacial de uma amostra de pontos, através da Função K, segue os seguintes passos:

1. Selecionar uma distância incremento $\left(d_{l a g}\right)$, também chamada de lag e calcular o número de lags $\left(n_{\text {lag }}\right)$ possíveis através da Equação 06;

$$
n_{\text {lag }}=\frac{D}{d_{\text {lag }}}
$$

onde $D$ é a maior distância entre pontos da amostra;

2. Definir $g=1$ como o número da iteração para iniciar o processo;

3. Para cada ponto da amostra, criar um buffer com o raio $h$ (Equação 07);

$$
h=d_{\text {lag }} \cdot g
$$

4. Para cada ponto, contar o número de pontos que caem dentro da área circular (vizinhança) de raio $h$; este contador é representado por $n(h)$; 


$$
n(h)=\sum_{i} \sum_{j} I_{h}\left(d_{i j}\right), i \neq j
$$

onde:

$d_{i j}$ : distância entre os pontos $i$ e $j$;

$I_{h}$ : é uma função indicadora. $I_{h}=1$ se $d_{i j}<\mathrm{h}$, caso contrário $I_{h}=0$.

5. Aumentar o raio da área circular em $d_{\text {lag }}$;

6. Repetir os passos 3, 4 e 5, aumentando $h$ até que o valor de $g$ seja igual ao de $n_{\text {lag }}$;

7. Calcular a função $K(h)$, pela Equação 9, e comparar com o padrão aleatório;

a. Se $K(h)$ for igual ao valor de $\pi h^{2}$, o padrão da amostra é aleatório;

b. Se $K(h)$ for menor que o valor de $\pi h^{2}$, o padrão da amostra é disperso;

c. Se $K(h)$ for maior que o valor de $\pi h^{2}$, o padrão da amostra é agrupado;

$$
K(h)=\frac{A}{n^{2}} \sum_{i} \sum_{j} I_{h}\left(d_{i j}\right)
$$

É possível ainda inferir sobre o padrão de distribuição utilizando o auxílio de um gráfico, onde o eixo das abscissas é referente às distâncias ( $h$ ou lag), e o eixo das ordenadas referente à função empírica $L(h)$, conforme a Equação 10. Valores acima da reta do padrão aleatório indicam padrão agrupado, enquanto valores abaixo indicam padrão disperso. Os padrões agrupado e disperso são considerados estatisticamente significantes se suas curvas ficarem fora do intervalo de confiança do padrão aleatório, conforme pode ser visto na Figura 3, regiões A e B. A região C, delimitada pelas linhas do intervalo de confiança, é considerada estatisticamente significante para o padrão aleatório.

$$
L(h)=\sqrt{\frac{K(h)}{\pi}}-h
$$

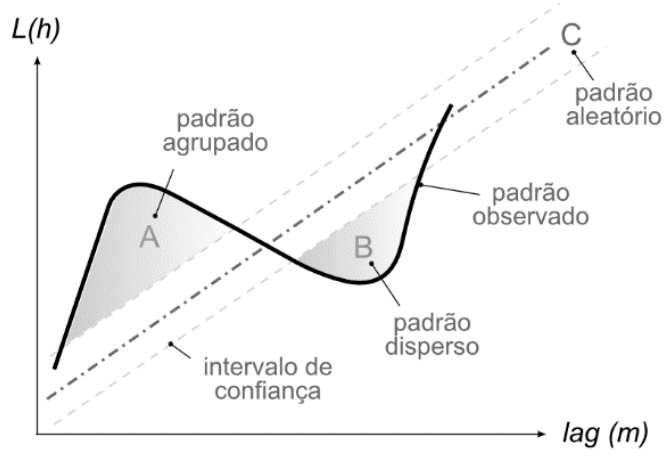

Figura 3: Gráfico gerado como resultado da aplicação da Função K.

Há a possibilidade de ver o quão significante ou não é o resultado obtido pela função $L(h)$. Entretanto, como o valor da análise da função $K$ inclui todos os pontos e distâncias entre os mesmos, ao contrário do vizinho mais próximo, não é necessário o uso do teste de hipótese. A função $K$ é mais complexa e menos intuitiva do que a análise de vizinho mais próximo. A aplicabilidade é limitada quando o processo espacial não é uniforme em todas as direções (anisotrópico), segundo Wong e Lee (2005). 


\section{Análise de tendências em dados espaciais}

Tradicionalmente, no controle de qualidade posicional em dados espaciais, para identificar a presença de efeitos sistemáticos utiliza-se da análise de tendências a partir do teste t de Student, conforme descrição em Merchant (1982), Ariza (2002), Nero (2005), Santos (2010).

Entretanto, o teste $t$ tem como premissa que a distribuição da amostra seja normal (Montgomery e Runger 2010). Contudo, há técnicas de estatística espacial que analisam a presença de tendência nos dados independentemente da distribuição estatística dos dados. Estas estatísticas espaciais são a Média Direcional e a Variância Circular (Wong e Lee, 2005).

O objetivo da Média Direcional é obter uma medida de tendência central da direção de um conjunto de vetores, cujas componentes são as discrepâncias nas ordenadas e abscissas.

De posse dos pontos de checagem e seus homólogos no dado espacial a ser analisado, traçam-se vetores ligando cada ponto de checagem com seus homólogos. A partir destes vetores, calcula-se a média direcional $\left(\theta_{R}\right)$ através da divisão do somatório do seno do azimute $\left(\theta_{v}\right)$ do vetor pelo somatório do cosseno do azimute, conforme a Equação (12).

$$
\tan \left(\theta_{R}\right)=\frac{\sum \sin \left(\theta_{v}\right)}{\sum \cos \left(\theta_{v}\right)}
$$

onde $\theta_{v}$ é o azimute do vetor na sua origem, ou seja, é o ângulo horário que se inicia no eixo de referência (Norte) até o alinhamento do vetor.

A Média Direcional $\left(\theta_{R}\right)$ apenas descreve uma tendência da direção, mas não consegue distinguir a variabilidade desta direção. Esta variabilidade é dada pela Variância Circular, que é calculada a partir do comprimento do vetor resultante $\left(C_{R}\right)$ determinado por:

$$
C_{R}=\sqrt{\left(\left[\sum \sin \left(\theta_{v}\right)\right]^{2}+\left[\sum \cos \left(\theta_{v}\right)\right]^{2}\right)}
$$

A Variância Circular $(S c)$ é obtida através do comprimento do vetor resultante e do número de vetores $(n)$, conforme a Equação (13). Para analisar se a média direcional é significativa ou não, deve-se analisar o resultado obtido pela variância circular. Se $S c$ for igual a zero $(S c=0)$, todos os vetores têm a mesma direção. Logo, a média direcional pode ser utilizada para representar a direção do conjunto de vetores. Mas se $S c$ for igual a 1 (um) $(S c=1)$, os vetores têm direções opostas, e, portanto, o valor da média direcional não é representativo da tendência da direção dos dados espaciais em questão.

$$
S c=1-\frac{C_{R}}{n}
$$

A Figura 4 ilustra a detecção de tendência com a aplicação da média direcional e variância circular. Na Figura 4.a a variância circular $(S c=0)$ detecta que a média direcional é representativa, indicando a presença de tendência nos vetores. Quando os sentidos dos vetores apresentam direções opostas $(S c=1)$, não é possível dizer que a média direcional é representativa (Figura 4.b). Já a Figura 4.c mostra uma situação onde a média direcional e a variância circular não detectam a tendência radial, que é uma tendência muito comum em fotogrametria. Como os vetores têm direções opostas ao centro da área de estudo, a variância circular será zero $(S c=1)$, não tendo sentido a análise da média direcional para este tipo de situação. 


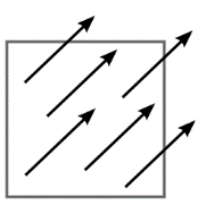

$\mathrm{Sc}=0$

(a)

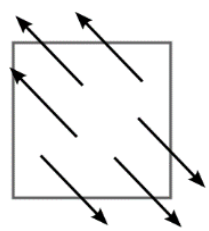

Sc $=1$

(b)

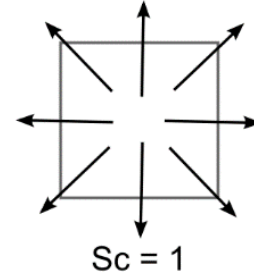

(c)

Figura 4: Análise de tendências utilizando a média direcional e a variância circular.

A análise de tendência na avaliação da acurácia posicional é de grande importância, já que estes efeitos sistemáticos podem ser modelados, minimizados ou corrigidos. Uma possível solução para minimizar/corrigir a tendência no produto cartográfico é realizar uma translação, de valor contrário às médias das discrepâncias posicionais $(\mathrm{x}, \mathrm{y}$ ou $\mathrm{z}) \mathrm{ou}$, para planimetria, uma translação de magnitude igual à média da discrepância resultante em sentido contrário da média direcional. Se forem realizadas correções no dado espacial, aplica-se uma nova avaliação. A princípio, não se descarta um dado espacial porque ele é tendencioso, já que este pode servir para a determinação de áreas, distâncias e ângulos entre feições, se a escala estiver consistente. Como já dito anteriormente, o principal foco do controle de qualidade é avaliar o conjunto de dados e dar um parecer para quais situações este pode ser aplicado.

\section{Padrão de acurácia posicional brasileiro}

O padrão brasileiro de acurácia posicional para dados espaciais é definido pelo Decreto-lei ${ }^{\circ}$ 89.817 de 1984, de acordo com as tolerâncias definidas no "Padrão de Exatidão Cartográfica" (PEC) e "Erro-Padrão" (EP). Tais tolerâncias tem seus valores definidos em função da escala de avaliação dos dados espaciais e das classes (A, B ou C) definidas por esse Decreto-lei. Em 2010, a Diretoria do Serviço Geográfico do Exército Brasileiro (DSG) publicou as Especificações Técnicas de Aquisição de Dados Geoespaciais Vetoriais (ET-ADGV), documento este ligado à Infraestrutura Nacional de Dados Espaciais (INDE) criada em 2008 pelo Decreto-lei ${ }^{\circ}$ 6.666. Em um de seus itens, a ET-ADGV explica como deve ser a aplicação do Decreto-lei $n^{\circ} .89 .817$ e cria uma classe mais restritiva destinadas para produtos cartográficos digitais (PEC-PCD).

Assim, para o enquadramento de um dado espacial em uma determinada escala e classe, segundo o padrão de acurácia posicional do Decreto-lei 89.817/ET-ADGV, devem ser atendidas duas condições (BRASIL, 1984; SANTOS, 2010; DSG, 2010):

i. Noventa por cento $(90 \%)$ dos pontos coletados no dado espacial, quando as suas coordenadas forem comparadas com as levantadas em campo, por método de alta precisão, ou levantadas em outro dado espacial de maior acurácia, deverão apresentar os valores de discrepâncias posicionais iguais ou inferiores ao valor da tolerância "PEC" em relação à escala e classe testada;

ii. O RMS (root mean square) da amostra de discrepâncias posicionais deve ser igual ou inferior à tolerância "EP" definido pela norma, para a escala e classe testada.

A Tabela 2 apresenta um resumo dos valores de PEC e EP definidos pelo padrão Decreto-lei 89.817/ET-ADGV. 
Tabela 2: Valores do PEC e do EP segundo Decreto-lei 89.817/ET-ADGV.

\begin{tabular}{c|c|c|c|c|c}
\hline Classe & Classe & \multicolumn{2}{|c|}{ Planimetria } & \multicolumn{2}{c}{ Altimetria } \\
\cline { 3 - 6 }$(\mathrm{PEC})$ & (PEC-PCD) & PEC & EP & PEC & EP \\
\hline- & $\mathrm{A}$ & $0,28 \mathrm{~mm}$ & $0,17 \mathrm{~mm}$ & 0,27 eq. & $1 / 6$ eq. \\
\hline $\mathrm{A}$ & $\mathrm{B}$ & $0,5 \mathrm{~mm}$ & $0,3 \mathrm{~mm}$ & $1 / 2$ eq. & $1 / 3$ eq. \\
\hline $\mathrm{B}$ & $\mathrm{C}$ & $0,8 \mathrm{~mm}$ & $0,5 \mathrm{~mm}$ & $3 / 5$ eq. & $2 / 5$ eq. \\
\hline $\mathrm{C}$ & $\mathrm{D}$ & $1,0 \mathrm{~mm}$ & $0,6 \mathrm{~mm}$ & $3 / 4$ eq. & $1 / 2$ eq. \\
\hline
\end{tabular}

onde "eq." significa equidistância da curva de nível e "esc." significa escala.

Um ponto de discussão sobre o padrão brasileiro é a adoção do termo "Exatidão". Este é definido como o grau de aderência do valor mais provável em relação ao valor verdadeiro. Nas ciências geodésicas e cartográficas um dos principais objetivos é a realização de posicionamentos para representar a forma da Terra. Sabe-se que qualquer medida realizada contém incertezas, não conhecendo o valor verdadeiro para um posicionamento ou localização na Terra. Existe sim, um valor mais provável para esta localização em questão, sendo obtido por instrumentos que possuam menores incertezas associados ao processo de posicionamento. Logo, é um equívoco usar o termo exatidão quando se tratar de qualidade posicional.

Segundo Monico et al. (2009) citando Mikhail e Ackerman (1976), a acurácia expressa o grau de proximidade de uma estimativa com o parâmetro para qual ela foi estimada, ou seja, o valor mais provável. Assim, a acurácia incorpora efeitos aleatórios e sistemáticos (Monico et al., 2009; Gemael, 1994). Portanto, entende-se que a acurácia envolve tanto a precisão (efeitos aleatórios) e a tendência (efeitos sistemáticos).

Sendo assim, este trabalho considera que um dado espacial é tido como acurado posicionalmente quando o mesmo for classificado no padrão Decreto-lei 89.817/ET-ADGV em um determinada escala e classe e ainda estar livre de efeitos sistemáticos em suas coordenadas, ou seja, não apresentar tendência.

\section{Metodologia proposta}

A metodologia proposta compreende basicamente os passos descritos na Figura 5. Na primeira parte da metodologia (Etapa 1), são obtidas informações a respeito do dado espacial a ser avaliado; escolha do número e local dos pontos; avaliação do padrão de distribuição espacial dos conjuntos de pontos utilizados para analisar a acurácia posicional e a partir do resultado obtido, a definição de qual primitiva gráfica (ponto, linha ou polígono) será utilizada no processo de avaliação da acurácia posicional.

Na segunda parte (Etapa 2), coletam-se os dados em campo; calculam-se as discrepâncias posicionais; e realizam-se as análises de tendência e precisão da amostra de discrepâncias, inferindo-se então sobre a acurácia do produto espacial. 
Etapa 1 - Análise da amostra de checagem

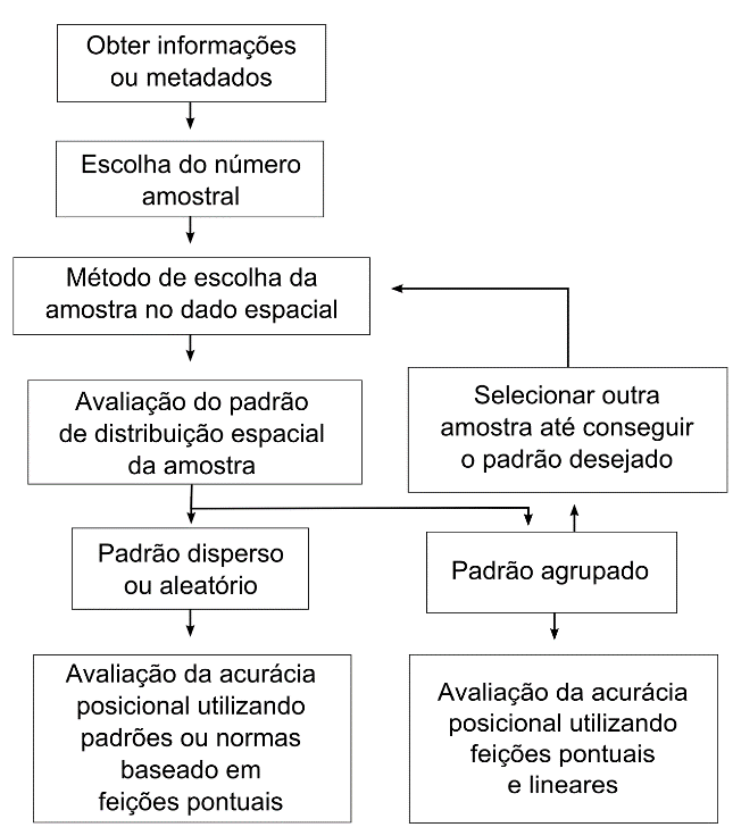

Etapa 2- Avaliação da Acurácia Posicional

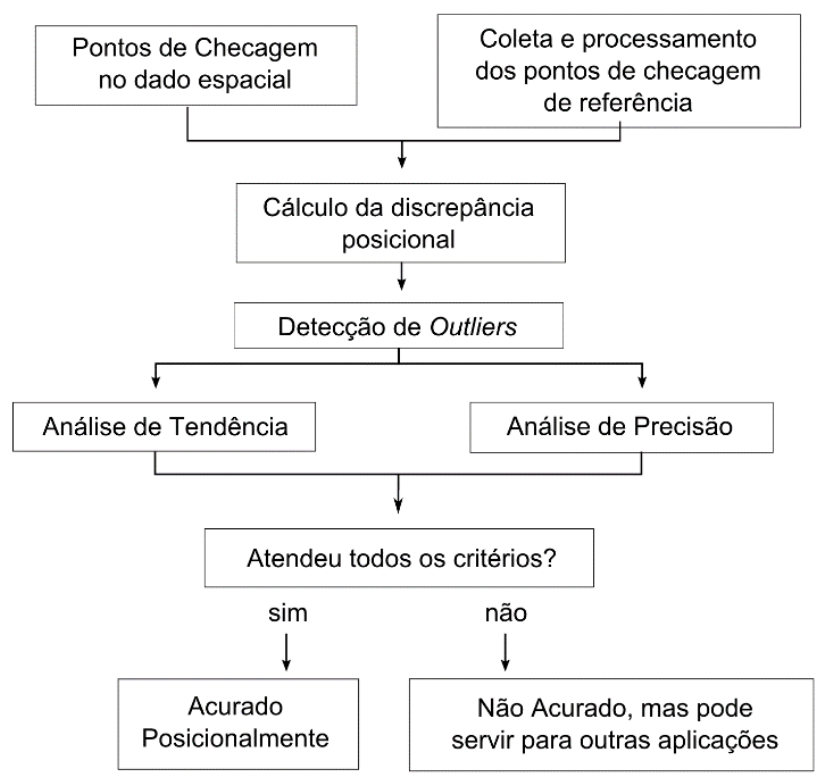

Figura 5: Fluxograma da metodologia proposta separada em duas etapas: (1) análise e planejamento da amostra de checagem para avaliação da acurácia posicional do dado espacial e; (2) o processo de avaliação da acurácia posicional, utilizando a análise de tendência e precisão.

O primeiro tópico na metodologia é a obtenção dos metadados ou o máximo de informações a respeito do dado espacial em avaliação, como sistema de projeção, datum, técnicas utilizadas em sua produção, etc. Esta informação é primordial para se ter uma ideia prévia sobre a escala a ser utilizada no processo de avaliação da acurácia posicional, assim como qual o nível de acurácia que os dados de referência (levantamento de campo ou um produto mais acurado) devem apresentar. Merchant (1982) sugere que o produto de referência utilizado para avaliação da qualidade posicional de um outro dado espacial deve ser $3 \mathrm{x}$ mais acurado. A norma STANAG 2215 da OTAN é mais rigorosa exigindo uma qualidade 5x maior do dado espacial utilizado como referência.

Em seguida, escolhe-se o tamanho da amostra (números de pontos de checagem). Trata-se de uma fase importante no processo de avaliação posicional, mas algumas normas não definem este número de pontos de checagem, a exemplo do padrão brasileiro. A norma americana NSSDA diz que cerca de 20 pontos distribuídos por toda área de abrangência do dado espacial são suficientes para avaliar um dado espacial; já a norma STANAG define um número de cerca de 168 pontos. Vários autores propõem alternativas para definição deste tamanho amostral, como Cintra e Nero (2015), Ariza e Atkinson (2008), Nogueira Jr. et al. (2004). Vale salientar que nesta etapa devese levar em conta a relação entre os custos econômicos para realizar o processo de avaliação da acurácia posicional e a confiabilidade dos resultados que serão obtidos.

Definido o número de pontos de checagem, passa-se para a etapa de escolha da localização de cada ponto de checagem no dado espacial. Em seguida, avalia-se o padrão de distribuição da amostra através de métodos descritos anteriormente na seção 2.

A maioria das normas de controle de qualidade posicional utiliza metodologias de avaliação baseadas em um conjunto de pontos de checagem que sejam bem definidos e distribuídos espacialmente sobre o dado espacial em avaliação, conforme apresentado na Figura 6.a. Assim, deve-se buscar atingir um padrão de distribuição dos pontos de checagem que seja disperso ou 
aleatório. Não satisfazendo o padrão de distribuição desejado, utiliza-se o artifício de mudar a localização de alguns pontos de checagem até obter o padrão desejável. Este processo de análise da amostra antes da coleta dos dados em campo otimiza o processo de avaliação da qualidade posicional em dados espaciais, evitando perda de tempo e diminuindo os custos na coleta de campo de outra amostra.

Entretanto, quando não se tem uma boa distribuição espacial de pontos de checagem (padrão agrupado), como apresentado na Figura 6.b, uma possível solução é avaliar a acurácia posicional do dado espacial através da integração de pontos e feições lineares, como apresentado na Figura 6.c, de modo a abranger toda a extensão do dado espacial. Maiores detalhes sobre a utilização de feições lineares no controle de qualidade posicional cartográfica podem ser vistos em Santos et al. (2015). Já na situação apresentada na Figura 6.d, não há como se proceder à avaliação de toda a área de estudo (representada pelo quadrado preto) baseando-se somente nos pontos agrupados em uma pequena porção da área de estudo. Nesta situação (6.d), deve-se realizar a avaliação da acurácia apenas na área de abrangência dos pontos de checagem (representada pelo fundo cinza). Outra solução para a situação de (6.b) e (6.d) pode ser o retorno às etapas anteriores (Figura 5), alterando o número ou a localização dos pontos de checagem até que tenha um padrão de distribuição espacial disperso ou aleatório.

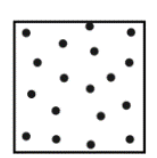

(a)

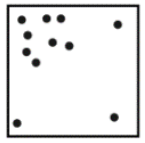

(b)

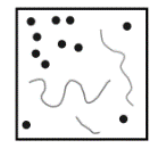

(c)

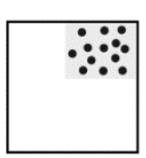

(d)

Figura 6: Distribuição de pontos de checagem para a avaliação da acurácia posicional.

Após analisar o padrão de distribuição espacial dos pontos de checagem, aplica-se a segunda parte da metodologia (Figura 5) para a avaliação da acurácia posicional do dado espacial. De posse dos pontos de checagem definidos no dado espacial em teste $(T)$ e seus homólogos coletados em campo (referência) ou de um produto cartográfico mais acurado $(R)$, calculam-se as discrepâncias posicionais planimétricas $\left(d_{2 D}\right)$, altimétricas $\left(d_{z}\right)$ ou tridimensionais $\left(d_{3 D}\right)$ (Equações 14, 15 e 16).

$$
\begin{gathered}
d_{2 D}=\sqrt{\left(\mathrm{X}_{\mathrm{T}}-\mathrm{X}_{R}\right)^{2}+\left(\mathrm{Y}_{\mathrm{T}}-\mathrm{Y}_{R}\right)^{2}} \\
d_{Z}=Z_{T}-Z_{R} \\
d_{3 D}=\sqrt{\left(\mathrm{X}_{\mathrm{T}}-\mathrm{X}_{R}\right)^{2}+\left(\mathrm{Y}_{\mathrm{T}}-\mathrm{Y}_{R}\right)^{2}+\left(\mathrm{Z}_{\mathrm{T}}-\mathrm{Z}_{R}\right)^{2}}
\end{gathered}
$$

Posteriormente, com os dados de discrepância posicional, realiza-se a análise de detecção de outliers. Esta detecção pode ser realizada utilizando o diagrama Boxplot, conforme Santos et al. (2015) ou utilizando a tolerância de $3 *$ EP, conforme sugerida por Santos (2010).

Vale ressaltar que vários autores na área de ciências cartográficas consideram sinônimos os termos outlier e erros grosseiros. Entretanto, segundo Melo e Castro (2013) e Amorim (2004) citando Gaspary (1987), outlier são resíduos ou discrepâncias que, estatisticamente, se diferenciam do conjunto de dados ao qual pertencem. Estes autores ainda afirmam que, geralmente, os outliers são causados por erros grosseiros. Assim, ao se detectar um outlier no controle de qualidade cartográfica, deve-se verificar se as feições foram bem identificadas no dado espacial de teste e de referência. Se esta identificação não estiver satisfatória (erro grosseiro), deve-se tentar corrigir ou então descartar tal ponto de checagem. Se a identificação 
estiver correta e mesmo assim a discrepância posicional for detectada como um outlier, entendese que o valor é representativo da qualidade posicional do dado espacial em avaliação, devendo tal ponto de checagem continuar na amostra de avaliação.

Em seguida, é feita a análise da tendência e da precisão dos dados. Na análise de tendência, pode-se utilizar o teste de t de Student ou a Média Direcional aliada com a Variância Circular; lembrando que o teste t de Student tem como necessidade que os dados sigam distribuição normal. $\mathrm{Na}$ análise de precisão aplica-se algum padrão de acurácia posicional, a exemplo o padrão brasileiro descrito pelo Decreto-lei 89.817/ET-ADGV. Finalizando, é realizada a junção dos resultados das análises de precisão e tendência para inferir sobre a acurácia posicional: se o resultado for preciso, atendendo a um padrão de qualidade, e não for tendencioso, pode-se concluir que o dado espacial é acurado posicionalmente; entretanto, se não for preciso e/ou for tendencioso, o dado espacial não é acurado posicionalmente. Vale salientar que se o produto for tendencioso, ou seja, caso haja um deslocamento posicional deste em relação à sua referência, pode-se facilmente aplicar uma translação no dado espacial (com o valor contrário da média da discrepância posicional) de forma a suprimir tal tendência nas coordenadas. Neste caso, sugerese realizar novamente a avaliação da acurácia posicional, já que realizando a translação, as coordenadas do dado espacial e, consequentemente, as discrepâncias posicionais irão se alterar, o que por sua vez, poderá influenciar o resultado da classificação quanto ao padrão do Decreto-lei 89.817/ET-ADGV.

\section{Experimento prático}

Para o experimento prático, avaliou-se a acurácia posicional de uma cena ortorretificada do sensor PRISM (Panchromatic Remote-sensing Instrument for Stereo Mapping) do satélite ALOS (Advanced Land Observing Satellite). Esta ortoimagem apresenta resolução espacial de 2,5 metros. A área de estudo compreende a extensão geográfica da cena da imagem desse sensor na região do município de Alvinópolis-MG, localizada entre as latitudes $19^{\circ} 58^{\prime} \mathrm{S}-20^{\circ} 18^{\prime} \mathrm{S}$ e longitudes $42^{\circ} 58^{\prime} \mathrm{W}-43^{\circ} 19^{\prime} \mathrm{W}$.

Para a definição do número da amostra de pontos de checagem, utilizou-se como parâmetro a norma norte-americana NSSDA, já que o padrão brasileiro não define um número de pontos de checagem. De posse da imagem ortorretificada, objeto da avaliação da acurácia posicional, definiram-se 26 pontos de checagem através de uma amostragem estratificada não-aleatória. A ortoimagem ALOS/PRISM cobre uma área de aproximadamente de $36,7 \mathrm{~km}$ x 35km. Então, para sistematizar a escolha dos pontos de checagem de modo que estes ficassem bem distribuídos na ortoimagem, gerou-se uma grade de $7 \mathrm{~km} \times 7 \mathrm{~km}$, obtendo um total de 36 quadrantes. Entretanto, somente 26 quadrantes serão efetivamente utilizados, devido à sobreposição destes quadrantes na ortoimagem (Figura 7).

Dentro de cada quadrante foi definido um ponto de checagem. As localizações destes pontos de checagem foram escolhidas conforme metodologia exposta em IBGE (2009), optando-se pela escolha de interseções de estradas rurais, conforme a Figura 7. 


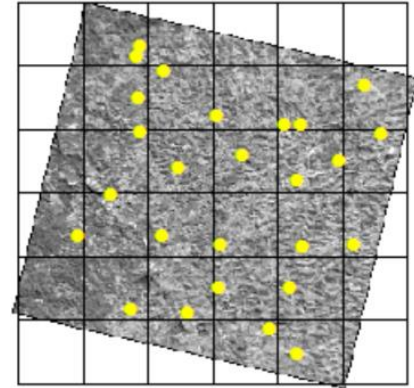

(a)

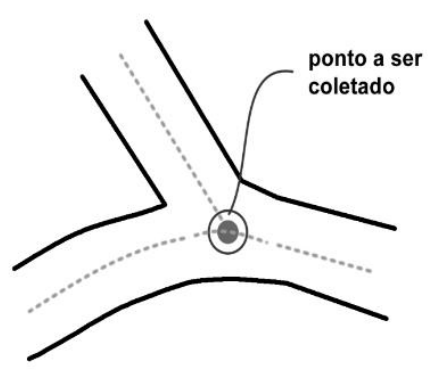

(b)

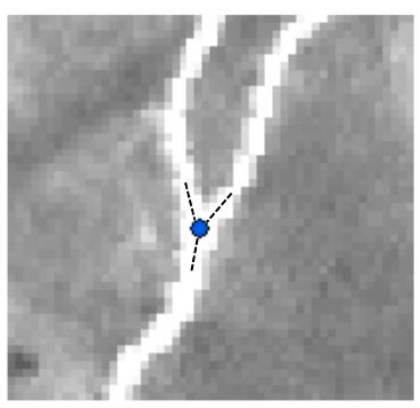

(c)

Figura 7: Distribuição espacial dos pontos de checagem (a) e metodologia utilizada para definição destes pontos na ortoimagem ALOS/PRISM (b) (c).

Alguns cuidados foram observados na escolha dos pontos, como: evitar locais com obstrução dos sinais dos satélites GNSS pela vegetação e/ou edificações nas imediações do ponto escolhido; evitar estradas muito movimentadas, já que a coleta dos pontos de checagem foi realizada no centro do cruzamento de estradas rurais; pontos com acesso facilitado, por questões de logística; observância do relevo, de modo que os pontos fossem distribuídos pelas altitudes baixas, médias e altas; escolher pontos bem distribuídos espacialmente.

Em seguida, de posse das coordenadas dos pontos de checagem planejados para avaliar a ortoimagem, aplicou-se a Função K e o método do Vizinho Mais Próximo de alta ordem para analisar o padrão de distribuição espacial dos pontos. A Tabela 3 apresenta os resultados obtidos para o Vizinho Mais Próximo utilizando a $1^{\mathrm{a}}, 2^{\mathrm{a}}$ e $3^{\mathrm{a}}$ ordem, de modo a analisar o padrão de distribuição espacial em escalas locais e regionais. A Figura 8 apresenta o gráfico resultante da aplicação do método da Função K, utilizando o software ArcGIS 10.2.

Tabela 3: Resultados da análise do padrão de distribuição espacial pelo método do vizinho mais próximo.

\begin{tabular}{c|c|c|c}
\hline \multirow{2}{*}{ Itens } & \multicolumn{3}{|c}{ Ordem } \\
\cline { 2 - 4 } & $1^{\mathrm{a}}$ & $2^{\mathrm{a}}$ & $3^{\mathrm{a}}$ \\
\hline Arrea $\left(\mathrm{km}^{2}\right)$ & \multicolumn{3}{|c}{1.277} \\
\hline$R_{\text {obs }}(\mathrm{m})$ & 4.384 & 6.063 & 7.298 \\
\hline$R_{\exp }(\mathrm{m})$ & 3.505 & 5.257 & 6.572 \\
\hline$R$ & 1,25 & 1,15 & 1,11 \\
\hline$Z_{\text {calc }}$ & 2,45 & 2,16 & 1,92 \\
\hline$Z_{\text {tab }}$ & 1,96 & 1,96 & 1,96 \\
\hline Padrão & Disperso & Disperso & Aleatório \\
\hline
\end{tabular}




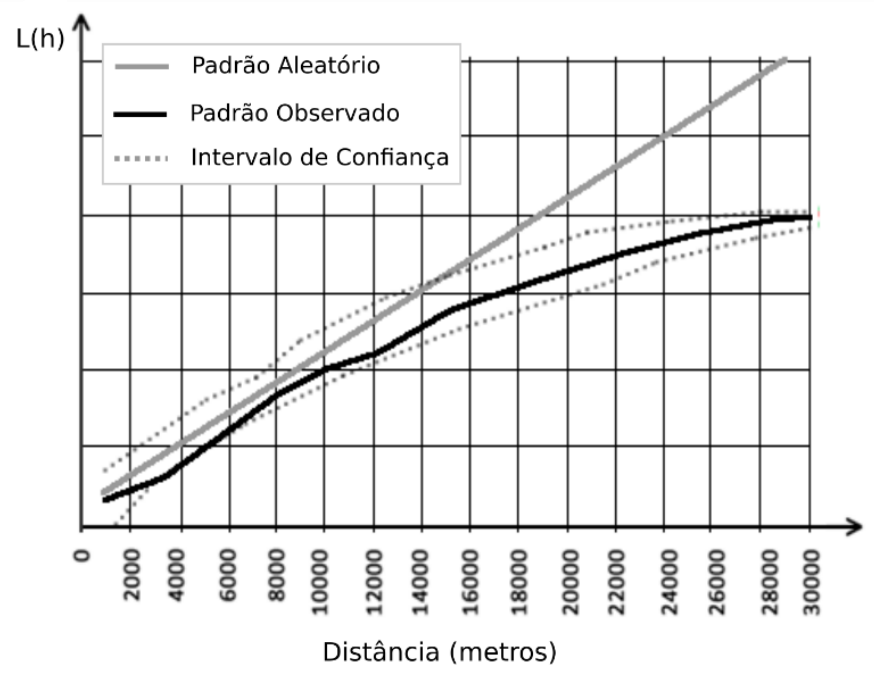

Figura 8: Gráfico resultante da aplicação da Função K para análise do padrão de distribuição espacial dos pontos de checagem.

No método do Vizinho Mais Próximo, todas as ordens testadas apresentaram o índice $\mathrm{R}$ maior que 1, sendo classificado, a princípio, como padrão disperso. Entretanto, deve-se verificar se este padrão é estatisticamente significante, razão pela qual se aplicou-se o teste $\mathrm{Z}$ utilizando um intervalo de confiança de $95 \%$. Para todas as ordens, exceto $3^{\mathrm{a}}$ ordem, como $Z_{\text {calc }}>Z_{\text {tab }}$, rejeitase a hipótese Ho que diz que a distribuição dos pontos segue um padrão aleatório, assim, o padrão de distribuição espacial dos pontos de checagem é disperso. Para a $3^{\mathrm{a}}$ ordem, como $Z_{\text {calc }}$ $<Z_{t a b}$, não se rejeita Ho sendo o padrão de distribuição espacial aleatório. Diante destes resultados, o método do Vizinho Mais Próximo evidencia que o padrão de distribuição espacial é localmente disperso e regionalmente aleatório, portanto, conclui-se o quão bem distribuído estão os pontos de checagem na área de estudo.

Aplicando o método da Função K, gera-se um gráfico conforme Figura 8, onde a linha cinza representa o padrão de aleatório teórico, a linha preta representa o padrão observado da amostra e as linhas pontilhadas representam o intervalo de confiança. Percebe-se que para uma distância pequena de até 4.000 metros, o padrão da amostra de checagem é disperso e estatisticamente significativo, já que a curva observada se encontra abaixo da curva de confiança da curva esperada; a partir desta distância o padrão é disperso, mas significante estatisticamente para o padrão aleatório, já que a curva observada está dentro do intervalo de confiança para o padrão aleatório. Com padrões dispersos e aleatórios, conclui-se que, através da Função K, os pontos de checagem estão bem distribuídos na área de estudo.

Percebe-se que os métodos utilizados para a detecção do padrão de distribuição espacial em dados pontuais (Vizinho Mais Próximo e Função K) apresentaram resultados similares, se mostrando uma boa ferramenta para evitar a subjetividade do analista ao julgar o padrão de distribuição espacial de uma amostra. O padrão de distribuição dos pontos de checagem mostrou-se condizentes com a análise visual.

Verificado que o padrão dos pontos de checagem possui uma boa distribuição espacial na área de estudo, partiu-se então para a coleta dos dados em campo. O levantamento de campo foi realizado com o receptor GNSS, utilizando o método estático com a linha de base variando entre 60 a $100 \mathrm{~km}$. Todos os pontos processados tiveram precisão posicional melhor que $20 \mathrm{~cm}$.

Coletados todos os pontos de checagem em campo e seus homólogos na ortoimagem, obtiveramse as discrepâncias posicionais planimétricas de cada ponto, conforme Equação 14. A Figura 9 
apresenta o gráfico das discrepâncias posicionais planimétricas observadas na ortoimagem avaliada.

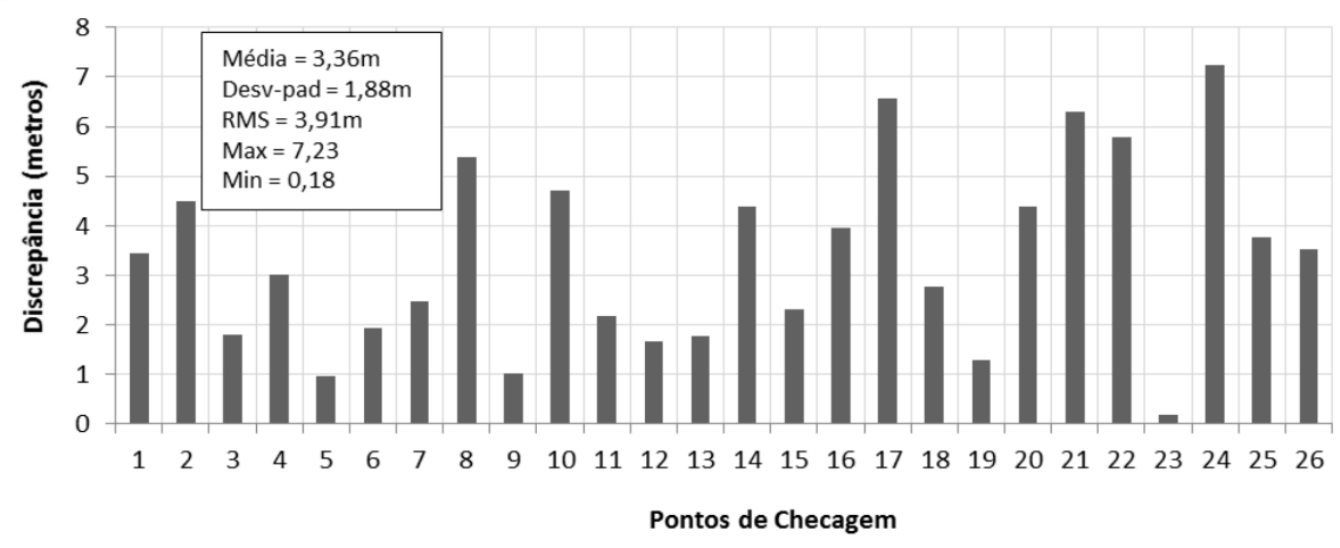

Figura 9: Discrepâncias planimétricas observadas na ortoimagem ALOS/PRISM.

De posse da amostra de discrepâncias posicionais planimétricas foi realizado a detecção de outliers pelo método do Boxplot, não sendo observada nenhuma discrepância posicional como outlier.

Após, pode-se aplicar as análises de tendência e precisão nos dados de checagem. Para avaliar a tendência da amostra de discrepâncias posicionais, aplicou-se um teste de normalidade ShapiroWilk ao nível de confiança de $95 \%$, utilizando o software $\mathrm{R}$, a fim de identificar qual método é passível de utilização. Os resultados evidenciam a não rejeição da hipótese nula $H o$, que diz que a amostra de discrepâncias segue distribuição normal. Assim, para a análise de tendência utilizou-se tanto o teste $t$ de Student quanto as estatísticas espaciais da Média Direcional / Variância Circular, conforme resultados apresentados na Tabela 4.

Tabela 4: Resultado da análise de tendência na ortoimagem ALOS/PRISM.

\begin{tabular}{c|c|c|c}
\hline \multicolumn{3}{c|}{ Estatística Inferencial } & \multirow{2}{*}{ Resultados } \\
\hline$t_{\text {calc } E}$ & $t_{\text {calc } N}$ & $t_{\text {tab }}$ & \\
\hline 0.13 & -1.4 & 1.7 & Não tendencioso \\
\hline \multicolumn{3}{c}{ Estatística Espacial } & \multirow{2}{*}{ Resultados } \\
\hline Média Dir. ${ }^{*}$ & \multicolumn{2}{|c|}{ Var. Circ. } & \\
\hline $206.20^{\circ}$ & \multicolumn{2}{|c|}{0.75} & Não tendencioso \\
\hline
\end{tabular}

* Média direcional calculada em relação ao eixo N (azimute). Orientação dos vetores: do ponto da imagem até o ponto de checagem em campo.

Como o valor absoluto de $t$ calculado foi menor que o valor de $t$ tabelado nas componentes Este e Norte, pode-se inferir, pelo teste $t$ de Student, que a ortoimagem não é afetada por efeitos sistemáticos. $\mathrm{Na}$ análise pela média direcional, a variância circular foi próxima de 1 , mostrando que os vetores de discrepâncias estão em direções opostas, inferindo-se, portanto, que não há a presença de efeitos sistemáticos no produto avaliado. Logo, ambos os resultados, obtidos tanto pelo teste $t$ de Student quanto pela Média Direcional / Variância Circular mostraram-se similares, não detectando a presença de tendência na ortoimagem. Vale ressaltar que as técnicas de estatística espacial citadas têm a vantagem de não necessitar que os dados sigam quaisquer tipos 
de distribuição de probabilidades, a exemplo a distribuição normal. A Figura 10 apresenta os vetores e um modelo das discrepâncias posicionais planimétricas observadas na ortoimagem ALOS/PRISM.

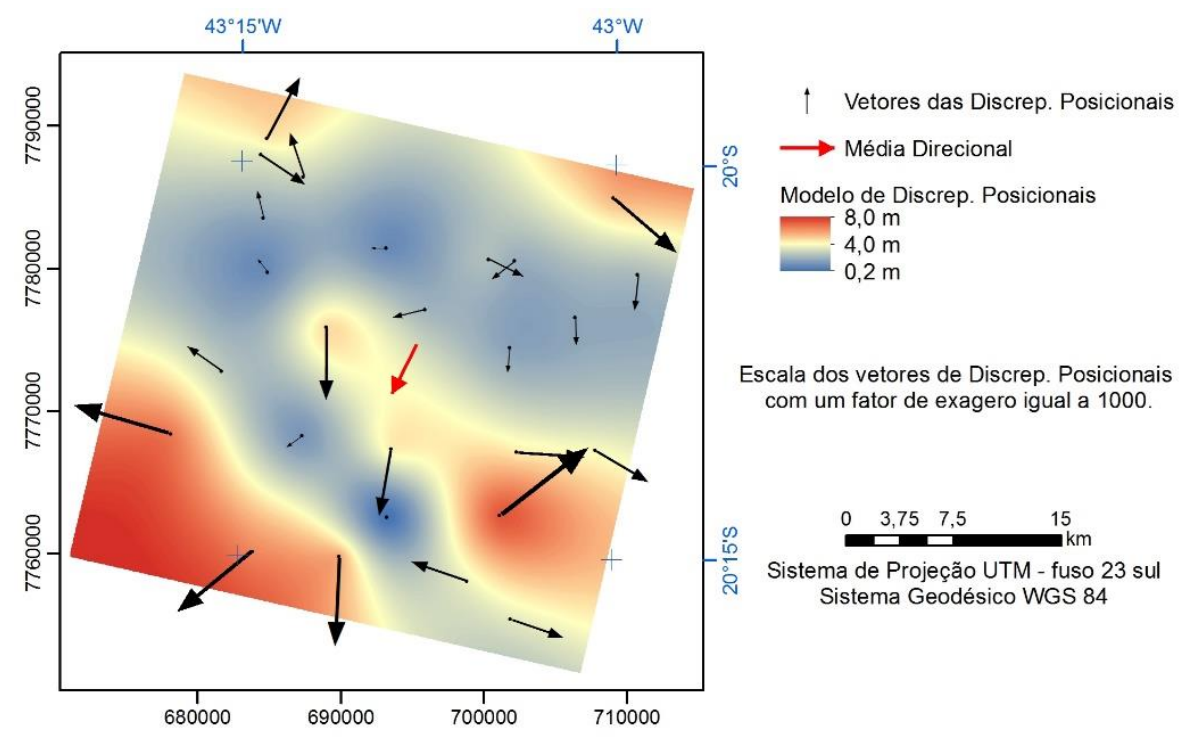

Figura10: Espacialização e vetores das discrepâncias posicionais planimétricas observadas na ortoimagem ALOS/PRISM.

Para o teste de precisão aplicou-se o padrão de acurácia posicional descrito no Decreto-lei ${ }^{\circ}$ 89.817/ET-ADGV, utilizando como referência a escala de 1:25.000, escala esta esperada para uma imagem com resolução espacial de 2,5 metros. Os resultados dos procedimentos utilizando o padrão brasileiro são apresentados na Tabela 5, indicando que a ortoimagem ALOS/PRISM atendeu às duas condições do Decreto-lei 89.817/ET-ADGV, para a escala de 1:25.000 na Classe A (PEC-PCD).

Tabela 5: Resultados da avaliação da acurácia posicional na ortoimagem.

\begin{tabular}{c|c}
\hline Itens & Resultado \\
\hline Media $\left(\mathrm{d}_{2 \mathrm{D}}\right)$ & $3,36 \mathrm{~m}$ \\
\hline RMS $\left(\mathrm{d}_{2 \mathrm{D}}\right)$ & $3,91 \mathrm{~m}$ \\
\hline Desvio-padrão $\left(\mathrm{d}_{2 \mathrm{D}}\right)$ & $1,88 \mathrm{~m}$ \\
\hline $\mathrm{n}^{\circ}$ da amostra $(\mathrm{n})$ & 26 \\
\hline Dado espacial é Tendencioso? & $N \tilde{A O}$ \\
\hline PEC-PCD $(1: 25.000$ - Classe A) & $7 \mathrm{~m}$ \\
\hline EP $(1: 25.000$ - Classe A) & $4,25 \mathrm{~m}$ \\
\hline \% de discrepâncias < PEC & $96,2 \%$ \\
\hline RMS < EP & SIM \\
\hline Dado espacial é Preciso ? & SIM \\
(Atende ao Decreto-lei 89.817/ET-ADGV) & \\
\hline Dado espacial é Acurado ? & SIM \\
\hline
\end{tabular}

Como a ortoimagem ALOS/PRISM atendeu aos padrões de precisão e tendência, conclui-se que a ortoimagem é acurada posicionalmente para a escala 1:25.000. 


\section{Considerações finais}

Ao longo deste trabalho, pôde-se perceber que é possível incorporar técnicas simples de estatística espacial para análise do padrão de distribuição espacial e detecção de tendências no processo do controle de qualidade posicional.

Percebeu-se que a análise do padrão de distribuição espacial de dados pontuais com as técnicas do Vizinho Mais Próximo e Função K podem ser adotadas em normas de acurácia posicional com o objetivo de eliminar a subjetividade do analista ao detectar como os dados estão distribuídos em toda a área de estudo. É importante ressaltar que um analista e/ou produtor experiente detecta com sucesso se os pontos utilizados estão bem distribuídos ou não, mas é importante a análise estatística para aperfeiçoar e proporcionar maior rigor a este processo, tornando-o menos intuitivo, mais técnico e científico.

A metodologia para avaliação da acurácia posicional proposta neste trabalho apresenta um significativo ganho com a abordagem do padrão de distribuição espacial da amostra de checagem no processo de avaliação do dado espacial, além da análise de tendências utilizando a estatística espacial da Média Direcional e Variância Circular.

Deve ficar claro que na avaliação da acurácia posicional em dados espaciais é essencial identificar as incoerências, o nível de precisão, além de classificar de acordo com um padrão. Como consequências da avaliação de acurácia posicional podem ser apontadas a identificação de algumas soluções para a minimização das incoerências do dado espacial em teste; indicação de qual a possível utilização deste em termos de extração de feições cartográficas e informações geométricas.

A avaliação da ortoimagem ALOS/PRISM apresentou bons resultados em termos de acurácia posicional, classificando a ortoimagem como classe A para a escala de 1:25.000. De um modo geral, as imagens ALOS/PRISM apresentam uma boa qualidade geométrica, conforme especificado pelo fabricante, tendo como principais aplicações as restituições cartográficas na escala de 1:25.000 e menores, sendo uma boa opção para a atualização do mapeamento sistemático brasileiro, já que este se encontra bastante defasado.

\section{REFERÊNCIAS BIBLIOGRÁFICAS}

Amorim, G. P. 2004. Confiabilidade de Rede GPS de Referência Cadastral Municipal - Estudo de Caso: Rede do Município de Vitória (ES). São Carlos, Brazil. Universidade de São Paulo.

Anjos, A., Mazza, M.C.M., Santos, A.C.M.C, and Delfini, L.T. 2004. “Análise Do Padrão de Distribuição Espacial Da Araucária (Araucaria Angustifolia) Em Algumas Áreas No Estado Do Paraná, Utilizando a Função K de Ripley." Scientia Florestalis 66: 38-45.

Arbia, G., Griffith, D., and Haining, R. 1998. "Error Propagation Modelling in Raster GIS: Overlay Operations." International Journal of Geographical Information Science 12 (2). Taylor \& Francis Group: 145-67.

Ariza, F.J. 2002. “Calidad En La Producción Cartográfica.” Ra-Ma.

Ariza, F.J., and Atkinson, A.D. 2008. "Analysis of Some Positional Accuracy Assessment Methodologies." Journal of Surveying Engineering 134 (2). American Society of Civil Engineers: 45-54. 
Aronoff, S. 1989. Geographic Information Systems: A Management Perspective. WDL Publications.

BRASIL. 1984. Decreto $\mathrm{N}^{\circ} 89.817$ de 20 de Junho de 1984. Normas Técnicas Da Cartografia Nacional. Brazil. http://www.planalto.gov.br/ccivil_03/decreto/1980-1989/D89817.htm.

Cintra, J.P., and Nero, M.A. 2015. "New Method for Positional Cartographic Quality Control in Digital Mapping." Journal of Surveying Engineering 141 (3). American Society of Civil Engineers.

Dalmaso, C.M., Inoue, M.T., Oliveira Filho, P.C., and Marcelino, V.R. 2013. "Padrões espaciais na regeneração de Ocotea odorifera na floresta nacional de Irati, PR.” Floresta 43 (2): 301.

DSG. 2011. "Especificação Técnica Para a Aquisição de Dados Geoespaciais Vetoriais (ETADGV).” Brasil.

Galo, M., and Camargo, P.O. 1994. “O Uso Do GPS No Controle de Qualidade de Cartas.” In $1^{\circ}$ Congresso Brasileiro de Cadastro Técnico Multifinalitário. Florianópolis, Brazil.

Gaspary, W.F. 1987. Concepts of network and deformation analysis. Monograph II. School of Surveying. University of New South Wales. Kensington, N. S. W. Australia.

Gemael, C. 1994. Introdução ao Ajustamento de Observações: aplicações geodésicas. Editora UFPR. Curitiba.

IBGE. 2009. "Avaliação Planialtimétrica de Dados ALOS/PRISM Estudo de Caso: Itaguaí - RJ." ISO. 2002. ISO 19113: Geographic Information - Quality Principles.

Jones, A.P., Langford, I.H., and Bentham, G. 1996. "The Application of K-Function Analysis to the Geographical Distribution of Road Traffic Accident Outcomes in Norfolk, England." Social Science \& Medicine (1982) 42 (6): 879-85.

Melo, D.C.R, and Castro, A.R. 2013. Uma Nova Abordagem Para Detecção de Outliers em Séries Temporais: Estudo de Caso em Consumo de Energia na Região Amazônica. In Proceeding Series of the Brazilian Society of Applied and Computational Mathematics, Vol. 1, $\mathrm{n}^{\circ} 1$.

Merchant, D.C. 1982. "Spacial Accuracy for Large Scale Line Maps." In Technical Congress of Surveying and Mapping, 222-31.

Monico, J.F.G, Dal Poz, A.L., Galo, M., Santos, M.C, and Oliveira, L.C. 2009. “Acurácia e precisão: revendo os conceitos de forma acurada." Boletim de Ciências Geodésicas 15 (3).

Montgomery, D.C., and Runger, G.C. 2010. Applied Statistics and Probability for Engineers.

Nero, M.A. 2005. "Propostas para p Controle de Qualidade de Bases Cartográficas com Ênfase na Componente Posicional.” São Paulo, Brazil: Escola Politécnica da USP, Brazil.

Nogueira Júnior, J.B., Monico J.F.G, and Tachibana, V.M. 2004. "Tamanho da amostra no controle de qualidade posicional de dados cartográficos." Boletim de Ciências Geodésicas.

Nogueira Júnior, J.B. 2003. "Controle de Qualidade de Produtos Cartográficos: Uma Proposta Metodológica." Presidente Prudente, Brazil: Faculdade de Ciências e Tecnologia - UNESP, Brazil.

Ripley, B.D. 1977. "Modeling Spatial Patterns." Journal of the Royal Statistical Society B 39 (2): $172-212$.

Santos, A.P. 2010. “Avaliação Da Acurácia Posicional Em Dados Espaciais Com O Uso de Estatística Espacial.” Viçosa, Brazil: Universidade Federal de Viçosa. 
Santos, A.P., Medeiros, N.G., Santos, G.R., and Rodrigues, D.D. 2016. "Avaliação da Acurácia Posicional Planimétrica em Modelos Digitais de Superfície com o uso de Feições Lineares." Boletim de Ciências Geodésicas.

Wong, D.W.S., and Lee, J. 2005. "Statistical Analysis of Geographic Information with ArcView GIS and ArcGIS." John Wiley \& Sons.

Recebido em setembro de 2015.

Aceito em fevereiro de 2016. 